\title{
Prescribing HIV PrEP and Education Needs Among Care Providers
}

\author{
Martin K. Logo \\ Walden University \\ Hadi Danawi \\ Walden University \\ Aimee Ferraro \\ Walden University
}

Preexposure prophylaxis (PrEP) is a biomedical intervention to prevent the spread of HIV. Underprescribing PrEP could increase the probability of HIV exposure among serodivergent couples/partners and people who do not practice safer sex. Previous research on PrEP did not assess the association between awareness of PrEP, years of experience of the providers, provider types, and the frequency of PrEP among physicians. The purpose of this study is to test the hypothesis that awareness of PrEP, years of experience, and provider types might be predictors of the frequency of PrEP among physicians. Precaution adoption process model and cross-sectional design were applied to survey 100 physicians. Kendall's b correlation test and Fisher's exact test showed that awareness was the primary barrier to prescribing PrEP at the providers' level. Higher awareness of PrEP and years of experience were associated with the frequency of PrEP prescription. However, there was an insignificant difference between provider types and the frequency of PrEP prescription. The findings support the notion that independent from their years of experience and specialty, the more physicians know about PrEP, the more they prescribe it. The results and recommendations contribute to positive social change by providing information to develop a comprehensive PrEP education curriculum for care providers. Our findings indicate that physicians need education and training to fully understand the potential for PrEP to reduce HIV transmission.

Keywords: preexposure prophylaxis (PrEP), HIV, precaution adoption process model (PAPM), serodivergent relationship, Truvada

This study was completed in partial fulfillment of the doctoral requirements in public health/community health at the Walden University by Martin K. Logo, under the direction of Hadi Danawi, PhD. An approval from the Walden University Institutional Review Board was obtained prior to the data collection. The entire study was reviewed by a review committee composed of Dr. Hadi Danawi, Committee Chairperson, Public Health Faculty; Dr. Aimee Ferraro, Committee Member, Public Health Faculty; and Dr. James Rohrer, University Reviewer, Public Health Faculty. Wiley Global has given the permission to adapt its precaution adoption process model algorithm to this study. 


\section{Introduction}

HIV is still among the top three public health concerns in the United States (Centers for Disease Control and Prevention [CDC], 2013). Despite the large amounts of money and steady effort put into research and prevention programs, the United Stated registers 50,000 new HIV cases yearly (CDC, 2013). HIV preexposure prophylaxis (PrEP) is a revolutionary, novel biomedical intervention in the last 6 years. PrEP regimen ${ }^{1}$ is recommended to HIV-negative people who might be at higher risk for the HIV infection like serodivergent couples/partners ${ }^{2}$ (AIDS.gov, 2014). For different reasons, it makes sense to use PrEP to prevent HIV (World Health Organization [WHO], 2013). For example, antiretroviral therapy has $96 \%$ efficacy against HIV transmission to the uninfected people who are at higher risk. These individuals include serodiscordant partners, men who have sex with men, needle-sharing drug users, and sex workers (CDC, 2013; Rosenthal et al., 2013).

However, Cairns (2013) reported that physicians do not prescribe PrEP very often in some geographic areas because they lack information about it. Even though some health insurance companies cover HIV PrEP (Horberg, 2012; Liu et al., 2014), physicians are reluctant to prescribe it (Krakower \& Mayer, 2013), but it is unclear why. In this study, we examined the role of HIV PrEP awareness, providers' years of service, and provider types as predictors of the frequency of $\operatorname{PrEP}$ prescription among care providers. A better understanding of the knowledge, behavior, and attitudes of physicians regarding the PrEP will better indicate where additional education may be needed.

\footnotetext{
${ }^{1}$ Understanding the PrEP regimen: PrEP is an HIV prevention regimen for HIV-free persons prone to ongoing substantial HIV exposure (Aids.gov, 2014). It consists of one pill of Truvada daily as a preventive measure to reduce HIV transmission risks (AIDSinfo, 2008). Truvada is made with $200 \mathrm{mg}$ of Emtricitabine and $300 \mathrm{mg}$ of tenofovir disoproxil fumarate. As an HIV nucleoside reverse transcriptase inhibitor drug, Truvada stops HIV from making copies. Its dosage for PrEP requires in a single pill (Choopanya et al., 2013). It can be (a) used with other antiretroviral drugs to treat HIV-positive patients and (b) used alone to prevent HIV infection in HIVnegative populations. When taken consistently, it has decreased the risk of HIV transmission among HIVnegative populations (Choopanya et al., 2013). Truvada is a component of PrEP that is a routine procedure. PreP regimen has four implementation phases. The first consists of assessing significant risk for HIV infection. The second is about establishing PrEP candidates' eligibility clinically (i.e., documented HIV-negative status, documented hepatitis B virus infection/immunization status, and checking for (a) no contraindicated medication, (b) no signs of severe HIV infection, and (c) healthy liver and good functioning kidneys). The third consists of prescribing Truvada (a steady oral dose of Truvada on the daily basis). The fourth is a follow-up. The follow-up services include every 3 months or 6 months for HIV and STI tests, counseling for medication adherence, renal function assessment, and pregnancy test for females, clean needles/syringes exchange, and substance abuse treatment services. The CDC recommended PrEP in addition to the following: Consistent and correct use of condoms,
}

- $\quad$ Getting tested periodically for HIV and sexually transmissible diseases,

- $\quad$ Adopting less risky sexual behaviors, and

- Using sterilized drug injection equipment or participating in a drug treatment program (Aids.gov, 2014).

${ }^{2}$ Serodivergent, also called serodiscordant, is associated with partners/couples with opposite HIV status (HIV negative versus HIV positive). 


\section{The Frequency and Challenges of PrEP Prescription}

Providers do not prescribe PrEP to their clients very often in the United States. Krakower and Mayer (2013) reported that only 4\% of participants had prescribed PrEP. Prescribing PrEP is challenging to some care providers.

According to Castillo (2013), the illiteracy about PrEP can lead to its underprescription. Studies focused on PrEP implementation challenges at the clients' level (Mansergh, Koblin, \& Sullivan, 2012) and barriers to free access to PrEP services (Puro, Palummieri, De Carli, Piselli, \& Ippolito, 2013).

\section{PrEP Education, Literacy Improvement Needs, and PrEP Guideline}

Education is the key strategy to enhance PrEP literacy. There was a significant relationship between awareness of PrEP and the clients' readiness to use PrEP (Young, Li, \& McDaid, 2013). The higher HIV infection incidence rate in the United States requires multilevel prevention approaches including PrEP education enhancement within care providers (Krakower \& Mayer, 2012).

Researchers at the University of California, Los Angeles showed that the 2014 PrEP guideline might overlook or underassess individuals who should be on PrEP (University of California-Los Angeles Health Sciences, 2017). It is necessary to develop a research-driven HIV-risk assessment tool to fill the gap.

\section{Need for Further Studies on PrEP: Workers and practitioners at the United Nations}

International Children's Emergency Fund, United Nations Program on HIV/AIDS, WHO, and CDC agreed that the time had come to promote universal access to prevention and treatment services (WHO, 2013). Detailed HIV PrEP guidelines need to be developed to improve physicians' readiness to provide PrEP and behavioral interventions to people at risk for HIV infection (Puro et al., 2013). That said, it is apparent that multisector studies of PrEP could provide insights to improve PrEP implementation, the gaps in PrEP prescription guideline, and the barriers to prescribe PrEP at the physicians level. Our research questions were as follows:

Research Question 1: What is the association between HIV PrEP awareness and the frequency of PrEP prescription among primary association between the number of years of service as a primary care provider or HIV specialist and the frequency of PrEP prescription among primary care providers and HIV specialists in the Quad Cities area?

Research Question 2: What is the association between the number of years of service as a primary care provider or HIV specialist and the frequency of PrEP prescription among primary care providers and HIV specialists in the Quad Cities area?

Research Question 3: What is the difference between provider types and the frequency of PrEP prescription among primary care providers and HIV specialists in the Quad Cities area? 


\section{Method}

The focus of this study was to explore HIV/AIDS health-protective behaviors by determining how primary care providers and HIV specialists make decisions to prescribe PrEP and how they translate their decisions into actions. For this reason, it was appropriate to adopt the precaution adoption process model (PAPM) as the theoretical framework to guide this study. We used the adapted visual representation (Figure 1) to explain the linkage between PAPM constructs and this study. Developed by Janis and Mann, PAPM defines the process in individuals' decisions making and the conversion of the decision into action in seven stages (Glanz, Rimer, \& Viswanath, 2008). Stage 1 is the unawareness of the health issue phase. In Stage 2, people learn about the problem for the first time but are not concerned about it. Stage 3, the decision-making phase, elucidates how individuals have concerns about health issues and start thinking about the appropriate responses. At this point, people can decide to stay at this stage or resolve to do nothing. This category of people falls under Stage 4, "halting" PAPM. The third possibility is to move to Stage 5 through precaution adoption. Stage 6, behavior initiation phase, allows people to act. Finally, Stage 7 is when people maintain their new behavior over time (Glanz, et al., 2008). PAPM allows inquirers to identify the stages that people go through when they start "health-protective behaviors" (Glanz, et al., 2008). It also provides indicators that help researchers to determine the factors favoring behavior change from one stage to another (e.g., from awareness to action). The model allowed us to identify barriers to PrEP provision at each stage.

We also applied Gollwitzer and Sheeran's (2006) implementation intentions concept to this study to examine how PrEP service implementation intentions can enhance the primary care providers and HIV specialists' willingness to prescribe it. The implementation intentions are a goal-directed behavior where people perform Behavior B if they met a Condition C (Gollwitzer \& Sheeran, 2006). The concept is effective in enhancing past behavior that leads to the prediction of new behavior (Orbeil, Hodgldns, \& Sheeran, 1997). We assumed that the concept will help primary care providers and HIV specialists to meet their PrEP prescription goals. The connection between the study variables and PAPM constructs is presented in Table 1. 

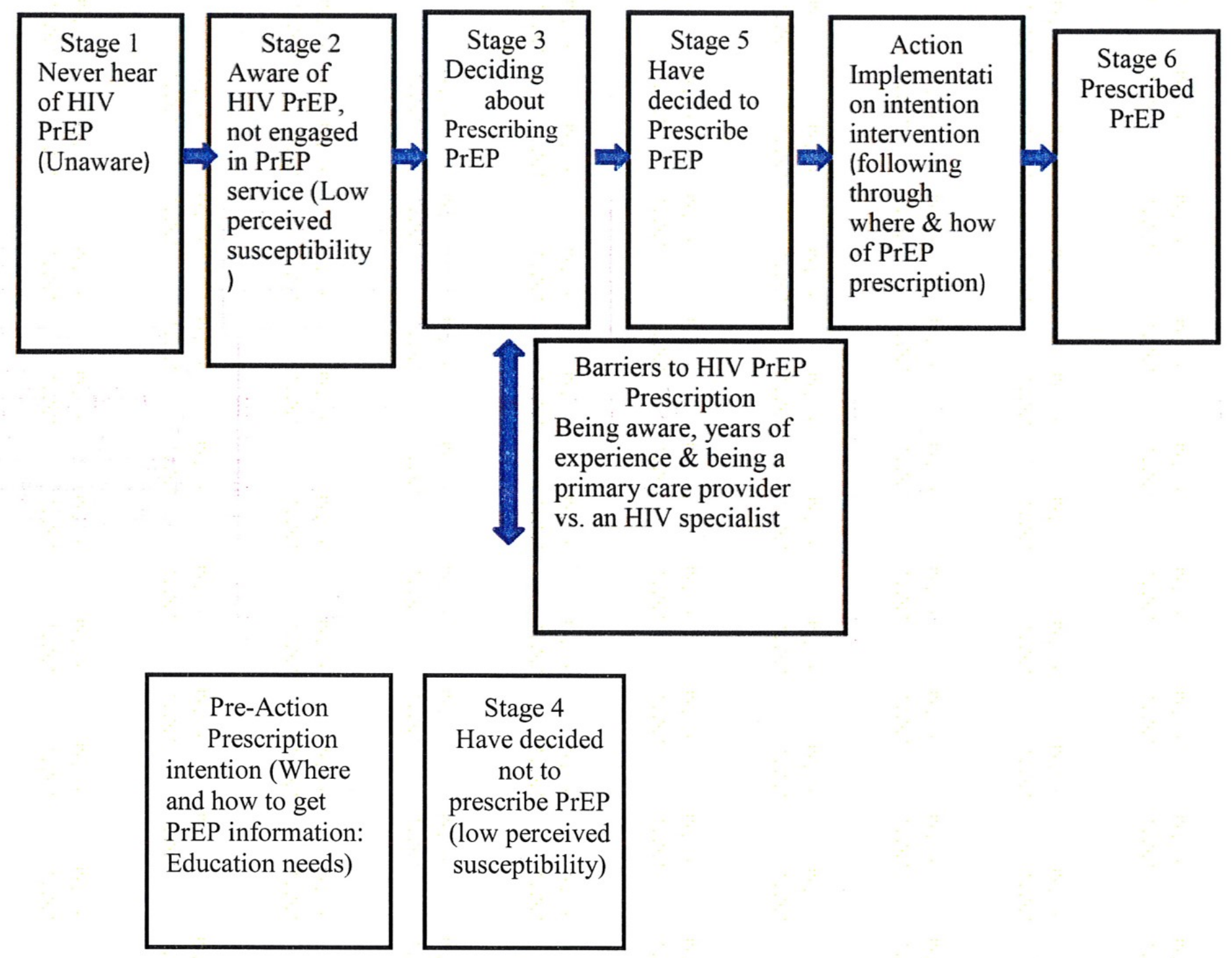

Figure 1. Visual Representation of Precaution Adoption Process Model Constructs Showing the Relationship Between Implementation Intentions Concept and the Gaps in HIV Preexposure Prophylaxis (PrEP) Prescription. Adapted From "Integrated Precaution Adoption Process Model and Implementation Intentions Concept Applied to Breast Cancer Screening," by K. K. Engelman and Colleagues (2011). Engaging Diverse, Underserved Communities to Bridge the Mammography Divide. Copyright 2011 by Engelman et al., Licensee BioMed Central Ltd. 
Table 1. Linkage Between Precaution Adoption Process Model (PAPM) Constructs and Study Variables $(N=100)$

\begin{tabular}{lccc}
\hline & \multicolumn{2}{c}{ Independent Variables } & \\
\cline { 2 - 4 } PAPM Constructs & $\begin{array}{c}\text { Preexposure } \\
\text { Prophylaxis (PrEP) } \\
\text { Awareness }\end{array}$ & Years of Service & $\begin{array}{c}\text { Difference in } \\
\text { Provider Type }\end{array}$ \\
\hline Stage 1: Unaware & $\begin{array}{c}\text { Never heard of } \\
\text { PrEP }\end{array}$ & As an indicator & As an indicator \\
Stage 2: Unengaged & $\begin{array}{c}\text { Never thought about } \\
\text { prescribing } \\
\text { PrEP }\end{array}$ & As an indicator & As an indicator \\
Stage 3: Undecided & $\begin{array}{c}\text { Undecided about } \\
\text { prescribing } \\
\text { PrEP }\end{array}$ & As an indicator & As an indicator \\
Stage 4: Do not want & $\begin{array}{c}\text { Do not want to } \\
\text { prescribe PrEP } \\
\text { to act }\end{array}$ & As an indicator & As an indicator \\
Stage 5: Decided to & $\begin{array}{c}\text { Plan to prescribe } \\
\text { act }\end{array}$ & As an indicator & As an indicator \\
Stage 6: Acting & $\begin{array}{c}\text { Prescribing } \\
\text { PrEP }\end{array}$ & As an indicator & As an indicator \\
Stage 7: & Prescribing \\
Maintenance & PrEP & As an indicator & As an indicator \\
\hline
\end{tabular}

Note. The outcome variable is the frequency of PrEP prescription.

Family practitioners, pediatricians, internists, obstetricians/gynecologists, and infectious disease/HIV specialists in the great Quad Cities area, up to a 70-mi radius from the city of Rock Island, Illinois, participated in the study. The cities include Davenport, Bettendorf, Clinton, De Witt, Wilton, Le Claire, Muscatine, Blue Grass, Eldridge, Orion, Durant, and Iowa City in Iowa and Rock Island, Milan, Silvis, East Moline, Moline, Alpha, Cambridge, Fulton, Cordova, Colona, Aledo, Coal Valley, and Peoria in Illinois. We sought the permission to conduct this study from the Walden University's Institutional Review Board.

Our recruitment effort consisted of using internet research tools to search for an updated list of primary care providers and HIV specialists practicing in the Quad Cities for May 15, 2015. We randomly selected 300 physicians among the 1,139 total physicians in the Quad Cities area.

We calculated a proportion of $26 \%$ (i.e., 300 divided by 1,139 times 100 ). We applied the $26 \%$ proportion to the total number of all Physicians in each of the five specialties in the area to determine the exact number of physicians to whom we should send the survey per specialty. Our final sample consisted of 97 family practitioners (26\% of 373$)$, 44 pediatricians ( $26 \%$ of 170$), 80$ internists (26\% of 308), 41 obstetricians/gynecologists ( $26 \%$ of 158), and 38 infectious disease/HIV specialists (26\% of 147 ) to whom we mailed the survey. We distributed the participants evenly across sex (150 females and 150 males) and across geographic location (i.e., 150 participants from Illinois and 150 from Iowa).

Our analytic sample was $n=100$ respondents (33.3\% response rate). We used SPSS and G*power analysis tools for the statistics tests. We designed our own survey instrument based on the modified Glanz et al. (2008) PAPM stage clarification algorithm (Table 2). Therefore, we pilot tested the 
instrument to ensure its fitness for the actual study. The pilot testing demonstrated appropriateness of the instrument.

Table 2. Modified Precaution Adoption Process Model (PAPM) Stage Algorithm to Assess Preexposure Prophylaxis (PrEP) Awareness

\begin{tabular}{lc}
\hline Questions and Answers & $\begin{array}{c}\text { Corresponding } \\
\text { PAPM Stage }\end{array}$ \\
\hline 1. Have you ever heard about HIV PrEP? & Stage 1 \\
No & \\
Yes (go to Question 3) & Stage 6 \\
2. Have you ever prescribed PrEP to a client? & \\
Yes & \\
No (go to Question 3) & \\
3. Which of the statements below describes better your opinions & \\
about prescribing PrEP? & Stage 2 \\
I have never thought about prescribing PrEP to clients. & Stage 3 \\
I am undecided about prescribing PrEP to clients. & Stage 4 \\
I am resolved to not prescribe PrEP to clients. & Stage 5 \\
I am resolved to prescribe PrEP to clients.
\end{tabular}

Note. Adapted with permission from "Precaution Adoption Process Model: Stage Classification Algorithm," by K. Glanz, B. K. Rimer, \& K. Viswanath, Health Behavior and Health Education: Theory, Research, and Practice (4th Ed.), p. 136. Copyright 2008 by John Wiley \& Sons, Inc.

Primary quantitative, categorical data including nominal and ordinal data were collected through the self-administered paper-based survey between October 24, 2015, and January 24, 2016. The United States' postal mailing system and in-person survey distribution strategy were used to distribute the questionnaires. A questionnaire cover letter and a stamped return envelope were included in the mailing. The study had one ordinal outcome (i.e., the frequency of HIV PrEP prescription) and two nominal independent variables (i.e., HIV PrEP awareness and the difference in provider's type), and one ordinal independent variable (i.e., the number of year of service). We used IBM SPSS Statistics 21 for all statistical analysis.

\section{Results}

\section{Descriptive Statistics}

There were $N=100$ valid responses and zero missing data. We evaluated the zip code of respondents to determine their geographic location (i.e., Illinois versus Iowa). The majority of participants were male and 45 to 54 years old. One quarter of them had 11-15 years of experience as a physician. The majority of respondents had very little awareness of PrEP. About 90\% of respondents had never prescribed PrEP or thought about prescribing PrEP to their clients either. Few participants declared that they know much about PrEP and its guidelines/protocol to prescribe or propose its services. For example, $87 \%$ of the respondents had low awareness about PrEP. Only 10\% of respondents had prescribed PrEP. The sample demographics presented Table 3. 
Table 3. Sample Demographics $(\mathrm{N}=100)$

\begin{tabular}{lcc}
\hline Variables & Frequency & Percent \\
\hline Sex of respondent & & \\
$\quad$ Male & 53 & $53 \%$ \\
$\quad$ Female & 47 & $47 \%$ \\
Age range of respondent & & \\
$25-34$ & 13 & $13 \%$ \\
$35-44$ & 21 & $21 \%$ \\
$45-54$ & 31 & $31 \%$ \\
$55-64$ & 28 & $28 \%$ \\
$65-74$ & 7 & $7 \%$ \\
Location of respondent & & \\
Illinois & 48 & $48 \%$ \\
Iowa & 52 & $52 \%$ \\
Year of experience of respondent & & \\
$0-5$ years & 14 & $14 \%$ \\
$6-10$ years & 16 & $16 \%$ \\
$11-15$ years & 20 & $20 \%$ \\
16-20 years & 14 & $14 \%$ \\
$21-25$ years & 18 & $18 \%$ \\
$26+$ years & 18 & $18 \%$ \\
Specialty & & \\
Family practitioners & 30 & $30 \%$ \\
Infectious disease/HIV specialists & 11 & $11 \%$ \\
Internists & 17 & $17 \%$ \\
Obstetricians/gynecologists & 19 & $19 \%$ \\
Pediatricians & 17 & $17 \%$ \\
\hline
\end{tabular}

\section{Main Analyses}

Two-sided Fisher's exact test revealed that the differences in the frequency of PrEP prescription across physicians' specialties were not statistically significant. There was a statistically significant association between years of experience and the frequency of PrEP prescription $(p=.002$, two-sided Fisher's exact test). Pearson chi-square test showed a significant association between awareness of $\operatorname{PrEP}$ and the frequency of PrEP prescription $(p=.017)$. The association between years of experience and the frequency of PrEP prescription was statistically significant too, confirming the result of Fisher's exact test performed earlier. Gender difference and geographic location covariates were not associated with the frequency of PrEP prescription.

Kendall's $\tau$ b correlation test showed a moderate, negative correlation between awareness of PrEP and the frequency of PrEP prescription that was statistically significant $(\tau b=-.367, p<.001)$. The frequency of PrEP prescription decreases. Kendall's $\tau \mathrm{b}$ correlation test also showed a weak negative correlation between years of service and the frequency of PrEP prescription that was statistically significant ( $\mathrm{cb}=-.228, p=.010)$. The frequency of PrEP prescription decreases when the number of years of service as a primary care provider decreases. Fisher's exact test of Research Question 3 showed no significant difference at the .05 significance level $(p=.130)$. 


\section{Discussion}

The results of the study provided information on the physicians' attitudes corresponding to each stage of the constructs of PAPM. For example, some participants are unaware of the issue. Some are aware but are unengaged. Others are undecided about taking action or decided not to act. Few of them have decided that they want to prescribe PrEP and will continue prescribing PrEP.

Based on our understanding of PrEP and PAPM, there are several interpretations of our findings.

\section{Stage 1: Unaware}

About one fifth of the physicians had never heard of PrEP. Therefore, they will never prescribe PrEP. This finding indicated the need for basic information (education) on PrEP for care providers. They will make best judgments about prescribing PrEP if they were PrEP literacy competent. They could then move to the next PAPM stages (i.e., unengaged, undecided, decided to act, or decided not to act).

\section{Stage 2: Unengaged}

About $70 \%$ of the participants learned very little or a little about PrEP. However, they were not yet engaged with the issue of under-prescribing PrEP. This finding is a call for formal education/training for the majority of physicians. It would allow HIV/AIDS prevention program developer to use training methods that make the PrEP issue and the need for action personally important to those unengaged physicians.

\section{Stage 3: Undecided}

About one quarter of the surveyed physicians were undecided about prescribing PrEP, meaning that they were engaged with the issue and looking for how to proceed. Because they did not yet form an opinion about prescribing PrEP, they would be less resilient to persuasion (DiClemente, Crosby, \& Kegler, 2002). The relevance of this finding is to remind potential PrEP providers about improving their PrEP literacy by exploring available PrEP provision protocol and guideline. For example, crosstraining among physicians within the same agency and coaching by PrEP experts through technical training is necessary for undecided physicians.

\section{Stage 4: Decided Not to Act}

Less than $5 \%$ of the surveyed physicians said that they do not want to prescribe PrEP. We assumed these people were aware of PrEP but have unexpressed reasons that challenge their decision to prescribe it. DiClemente et al. (2002) stated that those individuals might be difficult to persuade, and they ceased the PAPM process. On the other hand, some may hold off on deciding and stay undecided (Stage 3). This finding inspired the question about a suitable approach to engaging informed people who are refractory with prescribing PrEP. Proactively identifying these barriers to PrEP education programs would help health educators to use an alternative model to overcome them. 


\section{Stage 5: Decided to Act}

About $15 \%$ of the participants said that they plan to prescribe PrEP. One assumed that these individuals were conscious of the risks for not prescribing PrEP and the outcomes. Therefore, they decided to prescribe it. With this finding, researchers provided an empirical data as baseline information to measure the percentage of physicians who are ready to prescribe PrEP in the Quad Cities area as of October 2016.

\section{Stage 6: Acting}

Ten percent of the surveyed physicians are prescribing PrEP. Researchers also assumed that PrEP providers have some intrinsic and extrinsic motivations that were not examined in this study. Refreshment PrEP training is needed to empower that target population to continue prescribing PrEP.

\section{Stage 7: Maintenance}

The study did not provide relevant data to quantify the number of physicians who have maintained their prescribing of PrEP over time. Further investigation of this aspect of PAPM is desirable.

Providers' readiness to prescribe PrEP and patients' willingness to adopt it are the sine-qua-non conditions to promote PrEP regimen in the Quad Cities. However, researchers did not examine patients' attitudes toward, or need for, PrEP services in this study because the issue regarding prescribing PrEP in the Quad Cities was investigated at providers' level only.

The findings disconfirmed the assumption that the frequency of PrEP prescription is different as far as the physicians' specialty. Furthermore, the results did not support the hypothesis that there is a difference in provider types and the frequency of PrEP prescription among primary care providers and HIV specialists. Also, while Horberg and Raymond (2013) found that the high cost of PrEP could dissuade its prescribers and users, this study revealed that excessive cost and coverage of PrEP issues were not the primary barriers to prescribing it. Finally, the findings were opposite those of Puro et al. (2013), who demonstrated that only HIV/AIDS specialists had a privilege to prescribe PrEP. In fact, this study showed that even non-HIV/AIDS specialists (i.e., family practitioners, internists, obstetricians/gynecologists, and pediatricians) had prescribed PrEP.

The findings both confirmed and disconfirmed many results from the literature review on the concepts and key variables. For example, this study revealed the need for PrEP education and literacy improvement for physicians. Likewise, Krakower and Mayer (2012) showed that PrEP education enhancement within care providers is desirable to limit the higher HIV infection incidence rate in the United States.

\section{Limitations}

Response bias could arise from this study because of the self-reported survey. Although we strived to include more primary care physicians from different specialties, the physicians surveyed in this study did not represent a sample of all care providers who can prescribe PrEP in the United States. We aligned the questions strictly to the study's theoretical framework context to limit the response biases. Therefore, we are limited in the generalizability of the study. Future research could improve 
generalizability with a large sample size, including other medical specialties such as physician assistants and certified nurse practitioners.

\section{Recommendations}

To promote PrEP implementation and education, further implementation research is needed to understand and improve PrEP delivery at local and state levels. The approach is crucial to the better identification of, and response to, the gaps in PrEP prescription. Other calls for action include the necessity to

- develop a comprehensive PrEP education curriculum that aligns to the PAPM for care providers nationwide;

- include PrEP topics into the continued education online training modules for all physicians, physician assistants, and nurse practitioners;

- develop policies that support integrated PrEP implementation strategies (the strategies could consist of using HIV test to inform on PrEP, adding PrEP to risk assessment counseling process, integrating PrEP referrals into partner services, sexually transmitted disease clinics, and social network strategies);

- educate and train providers such as case managers, outreach staff, and testing counselors about PrEP guideline, PrEP protocols, its advantages and limits; and

- take PrEP information beyond care providers to the community at large through community forums, community outreaches, seminaries, peer education, and webinars.

\section{Positive Social Change Impact}

By empowering care providers to prescribe PrEP more often to vulnerable populations including persons in a serodiscordant relationship and others, the findings have potentials to bring positive changes to individuals and their families. The direct impacts include peace of mind and elimination of fear of the HIV infection. PrEP brings new dynamics (i.e., confidence, psychological supports, love, harmony, sexual freedom, etc.) in the family of serodiscordant individuals and also prevents new HIV infections. Providing PrEP to the professional sex workers and individuals with multiple sex partners will reduce HIV infection in the community. At the organizational level, it does contribute knowledge to the field of public health education. Further, this study presents PAPM as a potential evidence-based theoretical framework for future PrEP interventions.

\section{References}

AIDS.gov. (2014). Pre-exposure prophylaxis (PrEP). Retrieved from https://www.aids.gov/hiv-aidsbasics/prevention/reduce-your-risk/pre-exposure-prophylaxis/

AIDSinfo. (2008). Glossary of HIV/AIDS-related terms (6th edition). Retrieved from https://www.med.unc.edu/infdis/actu/files/GlossaryHIVrelatedTerms.pdf

Cairns, G. (2013). U.S. PrEP prescriptions more likely to go to southern women. aidsmap. Retrieved from http://www.aidsmap.com/US-PrEP-prescriptions-more-likely-to-go-to-Southernwomen/page/2755843/

Castillo, T. (2013). When doctors refuse to prescribe life-saving medication. Huffington Post. Retrieved from http://www.huffingtonpost.com/tessie-castillo/when-doctors-refusetopr_b_4136333.html 
Centers for Disease Control and Prevention (CDC). (2013). Update to interim guidance for preexposure prophylaxis (PrEP) for the prevention of HIV infection: PrEP for injecting drug users. Morbidity and Mortality Weekly Report, 62, 463-465. Retrieved from http://web.ebscohost.com.ezp.waldenulibrary.org/ehost/pdfviewer/pdfviewer?sid=bdbc28 b7cf47-48a8-9c3e-10b7a464274a\%40sessionmgr115\&vid=4\&hid=124

Choopanya, K., Martin, M., Suntharasamai, P., Sangkum, U., Mock, P. A., Leethochawalit, M., ... Vanichseni, S. (2013). Antiretroviral prophylaxis for HIV infection in injecting drug users in Bangkok, Thailand (the Bangkok Tenofovir Study): A randomized, double-blind, placebocontrolled phase 3 trial. Lancet, 381, 2083-2090. doi:10.1016/S0140-6736(13)61127-7

DiClemente, R. J., Crosby, R. A., \& Kegler, M. C. (2002). Emerging theories in health promotion practice and research: Strategies for improving public health. San Francisco, CA: JosseyBass.

Glanz, K., Rimer, B. K., \& Viswanath, K. (2008). Health behavior and health education: Theory, research, and practice (4th Ed.) San Francisco, CA: Jossey-Bass.

Gollwitzer, P. M., \& Sheeran, P. (2006). Implementation intentions and goal achievement: A metaanalysis of effects and processes. Advances in Experimental Social Psychology, 38, 69-119. doi:10.1016/S0065-2601(06)38002-1

Horberg, M. (2012). PrEP: Access and cost from private sector payor. Panel presentation from the TasP PrEP Evidence Summit: Controlling the HIV pandemic with antiretrovirals: Treatment as prevention and pre-exposure prophylaxis. London, UK: International Association of Providers of AIDS Care. Retrieved from www.iapac.org/tasp_prep/presentations/TPSlon12_Panel7_Horberg.pdf

Horberg, M., \& Raymond, B. (2013). Financial policy issues for HIV pre-exposure prophylaxis: cost and access to insurance. American Journal of Preventive Medicine, 44, S125-S128. doi:10.1016/j.amepre.2012.09.039

Krakower, D., \& Mayer, K. H. (2012). What primary care providers need to know about preexposure prophylaxis for HIV prevention: A narrative review. Annals of Internal Medicine, 157, 490497. doi:10.7326/0003-4819-157-7-201210020-00510

Krakower, D., \& Mayer, K. H. (2013). Engaging healthcare providers to implement HIV pre-exposure prophylaxis. Current Opinion in HIV AIDS, 7, 593-599. doi:10.1097/COH.0b013e3283590446

Liu, A., Cohen, S., Follansbee, S., Cohan, D., Weber, S., Sachdev, D., \& Buchbinder, S. (2014). Early experiences implementing pre-exposure prophylaxis (PrEP) for HIV prevention in San Francisco. PLoS Med, 11, e1001. doi:10.1371/journal.pmed.1001613

Mansergh G., Koblin, B. A., Sullivan, P. S. (2012). Challenges for HIV pre-exposure prophylaxis among men, who have sex with men in the United States. PLoS Med, 9, e1001286. doi:10.1371/journal.pmed.1001286

Orbeil, S., Hodgldns, S., \& Sheeran P. (1997). Implementation intentions and the theory of planned behavior. Personality and Social Psychology Bulletin, 23, 945-954. doi:10.1177/0146167297239004

Puro, V., Palummieri, A., De Carli, G., Piselli, P., \& Ippolito, G. (2013). Attitude towards antiretroviral pre-exposure prophylaxis (PrEP) prescription among HIV specialists. BMC Infectious Diseases, 13, 217. doi:10.1186/1471-2334-13-217 
Rosenthal, E., Piroth, L., Cua, E., Joulié, A., Ravaux, I., Chauveau, M., ... Cacoub, P. (2013). Preexposure prophylaxis (PrEP) of HIV infection in France: A nationwide cross-sectional study (PREVIC study). AIDS Care, 26, 176-185. Retrieved from http://www.tandfonline.com/doi/abs/10.1080/09540121.2013.803014

University of California-Los Angeles Health Sciences. (2017, January 10). CDC guidelines for HIV prevention regimen may not go far enough, study suggests: Risk calculator may fill that void. ScienceDaily. Retrieved from www.sciencedaily.com/releases/2017/01/170110094659.htm

World Health Organization (WHO). (2012). Guidance on oral pre-exposure prophylaxis (PrEP) for serodiscordant couples, men and transgender women who have sex with men at high risk of $H I V$ recommendations for use in the context of demonstration projects. Retrieved from http://apps.who.int/iris/bitstream/10665/75188/1/9789241503884_eng.pdf?ua=1

World Health Organization (WHO). (2013). Universal access to HIV/AIDS prevention, treatment, and care. Retrieved from http://www.who.int/hiv/topics/universalaccess/en/index.html

Young, I., Li, J., \& McDaid, L. (2013). Awareness and willingness to use HIV pre-exposure prophylaxis amongst gay and bisexual men in Scotland: Implications for biomedical HIV prevention. PLoS ONE, 8, 1-9. doi:10.1371/journal.pone.0064038

The Journal of Social, Behavioral, and Health Sciences is an open-access, peer-reviewed, online interdisciplinary journal focusing on research findings that address contemporary national and international issues. Its objectives are to (a) encourage dialogue between scholars and practitioners in the social, behavioral, and health sciences that fosters the integration of research with practice; (b) promote innovative models of interdisciplinary collaboration among the social, behavioral, and health sciences that address complex social problems; and (c) inform the relationship between practice and research in the social, behavioral, and health sciences.

Walden University Publishing: http://www.publishing.waldenu.edu 